\title{
Ten-year evolution of giant coronary artery aneurysms secondary to Kawasaki disease
}

\author{
Robert Sabiniewicz ${ }^{1}$, Lidia Woźniak ${ }^{1}$, Maksymilian Mielczarek ${ }^{2}$, \\ Dariusz Ciećwierz ${ }^{2}$, Rafał Pawlaczyk ${ }^{3}$ \\ ${ }^{1}$ Department of Pediatric Cardiology and Congenital Heart Diseases, Medical University of Gdansk, Poland \\ ${ }^{2}$ First Department of Cardiology, Medical University of Gdansk, Poland \\ ${ }^{3}$ Department of Cardiovascular Surgery, Medical University of Gdansk, Poland
}

\begin{abstract}
A 18-year-old male was referred to our hospital due to exercise angina of recent onset. Ten years prior to this the referral the patient had been diagnosed with Kawasaki disease (KD). At the time of diagnosis routine anti-inflammatory treatment for $\mathrm{KD}$, which consisted of intravenous gamma globulin $(2 \mathrm{~g} / \mathrm{kg})$ and high-dose aspirin $(80 \mathrm{mg} / \mathrm{kg})$ was administered. Echocardiography performed on the $10^{\text {th }}$ day of treatment showed aneurysmal dilatations of the left coronary artery (LCA). The patient was finally discharged with low-dose aspirin ( $5 \mathrm{mg} / \mathrm{kg}$ ). A coronary angiography scheduled 6 months later revealed multiple giant aneurysms of the LCA and occlusion of the right coronary artery (RCA). The dimensions of the largest aneurysm were approximately $15 \mathrm{~mm}$ $\times 15 \mathrm{~mm} \times 27 \mathrm{~mm}$ (Fig. $1 \mathrm{~A}-\mathrm{C})$. These angiographic findings required implementation of warfarin along with aspirin. Following treatment, the patient did not present with any symptoms for a decade.

On admission at our hospital, resting electrocardiogram (ECG) did not reveal any abnormalities and laboratory work up was within normal range (no elevation of troponins). The echocardiography showed normal left ventricle function. The exercise ECG stress test demonstrated profound ST-segment depression in leads I, aVL, and V4-V6. Subsequent coronary angiography revealed occlusions within heavily calcified aneurysms in the left anterior descending artery (LAD) and the obtuse marginal branch of the LCA, as well as lingering RCA occlusion (Fig. 1D-F) despite anticoagulant treatment. The patient was consequently referred for a surgical total arterial coronary revasculariza-
\end{abstract}

tion using bilateral internal mammary arteries Y-graft to the LAD and the posterior descending artery. The surgery was uneventful and resulted in complete symptoms resolution.

Kawasaki disease is an acute self-limiting childhood inflammatory disease associated with vasculitis, which predominantly affects coronary arteries. Cardiovascular manifestations are the leading cause of long-term morbidity and mortality. Coronary artery aneurysms (CAA) develop in $15-25 \%$ of untreated children. However, it is reduced to $7 \%$ when appropriate treatment is initiated within 10 days after fever onset [1]. CAA's were noted in $7.9 \%$ of patients younger than 40 years who underwent coronary angiography due to symptoms of myocardial ischemia, and in $85 \%$ of them history of KD was probable or definitive [2]. On the other hand, CAA frequently remains clinically silent for decades. Notwithstanding, they carry a lifelong risk for coronary artery thrombosis and myocardial ischemia or infarction. Lifelong aspirin is recommended in patients with $\mathrm{KD}$ who develop any kind of coronary artery dilatations. A combination of aspirin and oral anticoagulant should be recommended in patients with medium or giant CAA and those with a history of myocardial infarction [3]. The aforementioned case presents a well-documented natural history of $\mathrm{KD}$ complicated by giant CAA's and their subsequent occlusion. Knowledge of the unique therapeutic challenges associated with $\mathrm{KD}$ is of great importance for the physicians who may come across young adults complaining of chest pain.

Address for correspondence: Lidia Woźniak, MD, Department of Pediatric Cardiology and Congenital Heart Diseases, Medical University of Gdansk, ul. Dębinki 7, 80-211 Gdańsk, Poland, tel: +4858 34928 70, fax: +48 583492895 , e-mail: lidiawozniak@yahoo.com

Received: 28.04.2016 Accepted: 6.06.2016 


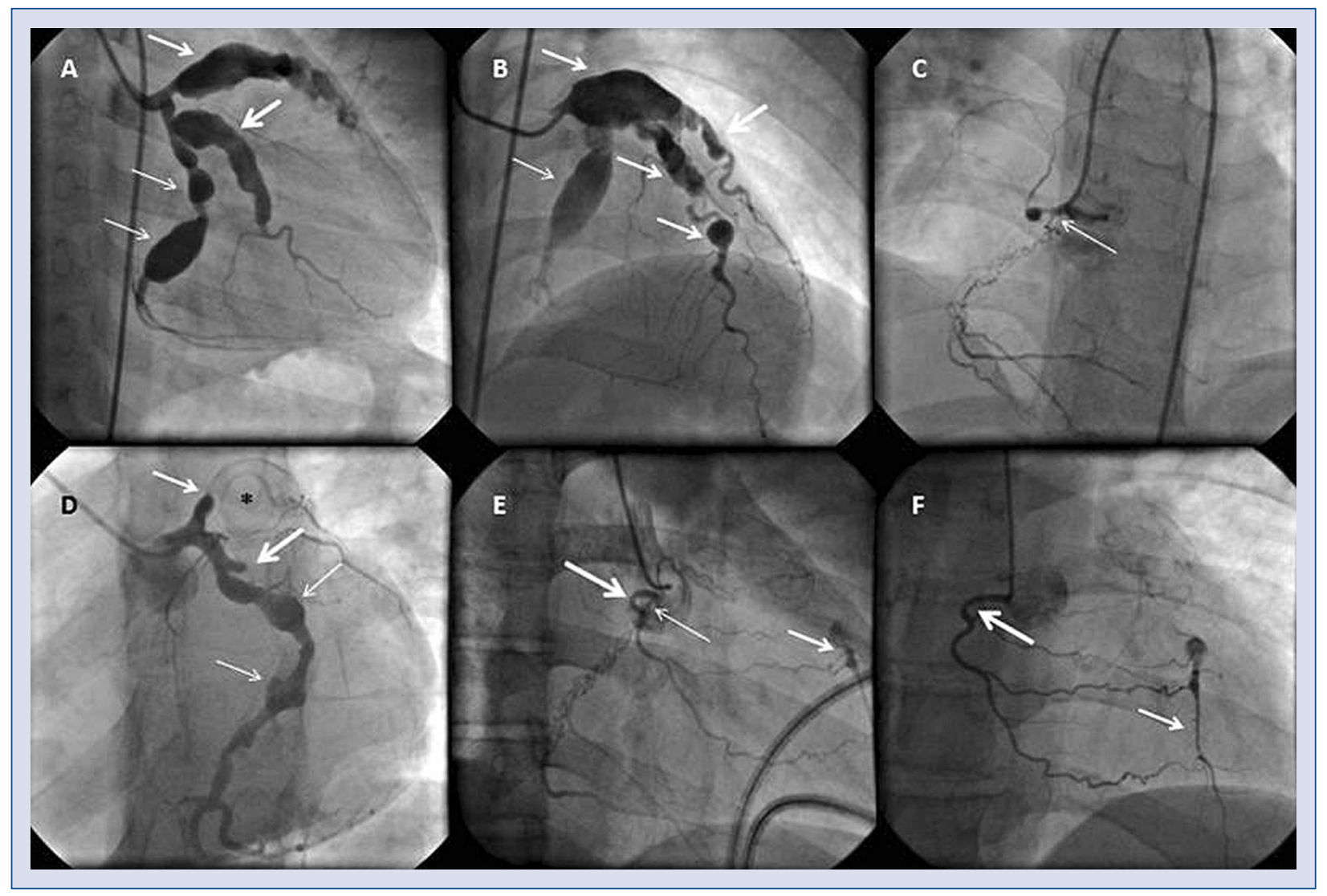

Figure 1. A, B. First angiography, left coronary artery (LCA): aneurysms (arrows) of the left anterior descending artery (LAD), aneurysm (thick arrow) of the obtuse marginal branch (OM), and aneurysms (thin arrows) of the continuous branch of the left circumflex branch (LCX); C. First angiography, right coronary artery (RCA): proximal RCA occlusion (thin arrow) with developed collateral vasculature; D. Second angiography, LCA: proximal occlusion of the LAD (arrow) with aneurysm cavity completely filled with partially calcified thrombus (asterisk); occlusion of the OM at the site of previous aneurysm (thick arrow) and patent continuous branch of the LCx with aneurysms partially filled with thrombus (thin arrows); E, F. Second angiography, RCA: lingering RCA occlusion (thin arrow) beyond takeoff of the acute marginal branch (thick arrow), which supplies via collaterals the distal part of the occluded LAD (arrow).

\section{References}

1. Shah V, Christov G, Mukasa T et al. Cardiovascular status after Kawasaki disease in the UK. Heart, 2015; 101: 1646-1655.

2. Rizk SR, El Said G, Daniels LB et al. Acute myocardial ischemia in adults secondary to missed Kawasaki disease in childhood. Am J Cardiol, 2015; 115: 423-427.
3. JCS Joint Working Group. Guidelines for diagnosis and management of cardiovascular sequelae in Kawasaki disease (JCS 2013). Circ J, 2014; 78: 2521-2562.

Conflict of interest: None declared 